\title{
EDITORIAL
}

\section{Inherent value of baseline measures when assessing the trajectory of health-related quality of life among children surviving critical illness}

\author{
Elizabeth Y. Killien ${ }^{1,2^{*}}$, R. Scott Watson ${ }^{1,3}$ and Jerry J. Zimmerman ${ }^{1,4}$
}

(C) 2018 Springer-Verlag GmbH Germany, part of Springer Nature and ESICM

As mortality in most pediatric intensive care units (PICUs) in developed countries has fallen to below $3 \%$ [1], there is growing awareness that children who survive critical illness remain at risk for long-term morbidity [2]. Correspondingly, there is emerging emphasis on including post-discharge status as a sensitive, clinically meaningful, and patient-centered outcome measure in pediatric critical care research [3], especially among populations where mortality rates are low $[4,5]$. Healthrelated quality of life (HRQL) incorporates multiple dimensions of potential morbidity into a single comprehensive measure and has been identified by both families and healthcare professionals as the most important outcome to assess among PICU survivors [5]. While many studies have demonstrated impairments in HRQL following pediatric critical illness [6-11], there remains a paucity of data describing which factors contribute most to HRQL decline and how HRQL changes over time.

In this issue of Intensive Care Medicine, Kyösti and colleagues report the HRQL and health status of over 1000 children and young adults in Finland 6 years after an ICU stay [12]. In a novel approach, the authors used a national ICU registry to identify all patients less than 18 years old admitted from 2009 to 2010 to any ICU in the country that treats children, resulting in a nationally representative cohort of over 3500 patients from 25 different

\footnotetext{
*Correspondence: elizabeth.killien@seattlechildrens.org ${ }^{1}$ Division of Pediatric Critical Care Medicine, Department of Pediatrics, Seattle Children's Hospital and The University of Washington School of Medicine, FA2.112, 4800 Sandpoint Way NE, Seattle, WA 98105, USA Full author information is available at the end of the article
}

facilities. They contacted families 6 years after the index ICU stay to assess their HRQL using the Pediatric Quality of Life Inventory (PedsQL ${ }^{\mathrm{TM}}$ ) and the $15 / 16 / 17 \mathrm{D}$ instrument commonly used in Finland. They defined their primary outcome of "poor HRQL" as a PedsQL ${ }^{\mathrm{TM}}$ score more than two standard deviations (SDs) below the published population mean. They received responses from $30 \%(n=1109)$ of surviving patients and found that $8.4 \%$ of respondents met their definition for poor HRQL, concluding that "the long-term quality of life after pediatric intensive care is good for the majority of children."

The authors are to be commended for what is among the largest studies to date evaluating HRQL following pediatric critical illness, with a national cohort representing a wide spectrum of admission diagnoses and a long follow-up period. What this work highlights, however, is how imperative it is to control for baseline health status or assess change from baseline HRQL when evaluating quality of life after illness. By comparing HRQL scores at a single time point to population means, the contribution of critical illness to poor HRQL is difficult to assess. Given a population mean PedsQL ${ }^{\mathrm{TM}}$ score of $81.4 / 100$ points [13], the approach utilized by the authors likely underestimates the burden of critical illness for patients whose baseline was above the mean and experienced substantial declines following their hospitalization, and overestimates it for others who started well below the mean but did not decline further (Fig. 1).

The impact of critical illness on subsequent HRQL may also have been underestimated by the authors' conservative definition of "poor" HRQL. Two SDs of the PedsQL ${ }^{\mathrm{TM}}$ mean is 31.8 points, which is seven times the

\section{Springer}




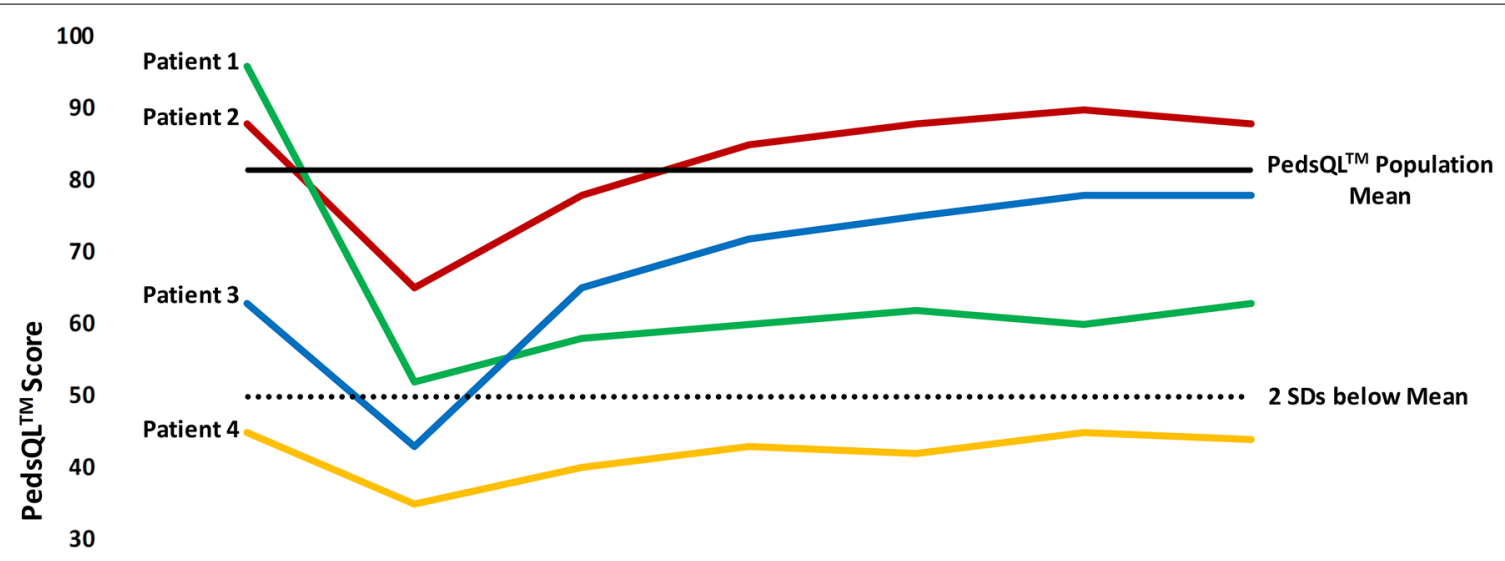

20

10

0

Baseline Admission Discharge 6 months 1 year 3 years 5 years
Time of Assessment

Patient 1: Previously healthy patient who sustains a traumatic brain injury and has persistent clinically significant HRQL deterioration from baseline but does not fall $>2$ SDs below the population mean

Patient 2: Previously healthy patient admitted with sepsis who recovers to baseline within 6-12 months of discharge

Patient 3: Patient with congenital heart disease who undergoes corrective cardiac surgery and has subsequent improvement in $H R Q L$ from ba seline

Patient 4: Patient with cerebral palsy admitted with respiratory illness who recovers back to baseline but remains $>2$ SDs below the population mean

Fig. 1 Theoretical health-related quality of life trajectory of sample patients following critical illness. SD standard deviation; HRQL health-related quality of life

minimum clinically significant difference of 4.5 points for this instrument [13] and results in a total follow-up score below 50/100. This represents extremely impaired HRQL; there may have been many patients with clinically significant deterioration not captured by this approach. Additionally, patients who responded were more likely than non-responders to have been admitted for elective surgical procedures, including $18 \%$ for cardiac surgery; these procedures may have actually contributed to improved HRQL postoperatively. In contrast, non-responders were more likely to have been admitted emergently with sepsis, injury, or metabolic problems. The sample thus likely represents a group with more potential for longterm recovery after hospitalization than the population as a whole. Finally, the overall burden of critical illness on survivor outcomes is notably underestimated by not acknowledging post-discharge mortality; in this cohort, more patients died after discharge than during their index hospitalization.

Of the factors associated with poor HRQL at follow-up, the most notable are the presence of chronic illness and ongoing healthcare needs. Nearly $95 \%$ of patients with low HRQL at follow-up had chronic conditions, most commonly neurologic diseases such as cerebral palsy and epilepsy, chromosomal abnormalities, and malignancies. Nearly all required healthcare services, and threequarters required daily medications. What is essential to understand, however, is whether these chronic diagnoses and healthcare needs were present prior to ICU admission 6 years prior, resulted from the critical illness, or developed in the intervening 6 years. While we agree with the authors that patients with chronic comorbidities may be particularly vulnerable to adverse outcomes after critical illness, comparing their HRQL to population means does not provide information about the trajectory of their HRQL after hospitalization. Children with cerebral palsy, for example, have a mean PedsQL ${ }^{\mathrm{TM}}$ score of 51.3 points [14] and thus many would meet the authors' criteria for poor HRQL even before an ICU stay.

The limitations of this study call attention to crucial gaps in the state of knowledge in this field, and highlight the urgent need to better understand the trajectory of HRQL following critical illness. The authors' use of a national ICU database is an intriguing demonstration of how such a resource might be able to be enhanced and adopted throughout health systems worldwide to address 
these questions. We recommend that a minimum pediatric ICU database should include patient demographics, baseline assessment of function and chronic illness (e.g., Functional Status Scale and Pediatric Medical Complexity Algorithm [15]), detailed information on admission diagnoses, an illness severity measure (e.g., PRISM or PIM), serial organ dysfunction assessment (e.g., daily PELOD), surgical procedures and hospital complications, ICU and hospital length of stay, and discharge Functional Status Scale. Additionally, we believe that incorporation of a baseline HRQL assessment is both feasible and expedient. The PedsQL ${ }^{\mathrm{TM}}$, for example, consists of only 23 questions and is typically completed in under $5 \mathrm{~min}$. Routine collection of premorbid health status, hospitalization data, and baseline HRQL would allow substantial improvements in our ability to determine change from baseline status across populations, better understanding of the actual causes of impaired HRQL, and identification of factors associated with recovery. Ultimately this information can be utilized to design interventional trials to improve long-term outcomes for children surviving critical illness.

\section{Author details \\ ${ }^{1}$ Division of Pediatric Critical Care Medicine, Department of Pediatrics, Seattle Children's Hospital and The University of Washington School of Medicine, FA2.112, 4800 Sandpoint Way NE, Seattle, WA 98105, USA. ${ }^{2}$ Harborview Injury Prevention and Research Center, University of Washington, Seattle, WA, USA. \\ ${ }^{3}$ Center for Child Health, Behavior, and Development, Seattle Children's Research Institute, Seattle, WA, USA. ${ }^{4}$ Center for Clinical and Translational Research, Seattle Children's Research Institute, Seattle, WA, USA.}

\section{Compliance with ethical standards}

Conflicts of interest

The authors have no conflicts of interest to disclose.

Received: 3 August 2018 Accepted: 21 September 2018 Published online: 28 September 2018

\section{References}

1. Burns JP, Sellers DE, Meyer EC, Lewis-Newby M, Truog RD (2014) Epidemiology of death in the pediatric intensive care unit at five U.S. teaching hospitals. Crit Care Med 42:2101-2108
2. Watson RS, Choong K, Colville G, Crow S, Dervan LA, Hopkins RO, Knoester H, Pollack MM, Rennick J, Curley MAQ (2018) Life after critical illness in children - toward an understanding of pediatric post-intensive care syndrome. J Pediatr 198:16-24

3. Aspesberro F, Mangione-Smith R, Zimmerman JJ (2015) Health-related quality of life following pediatric critical illness. Intensive Care Med 41:1235-1246

4. Menon K, McNally JD, Zimmerman JJ, Agus MS, O'Hearn K, Watson RS, Wong HR, Duffett M, Wypij D, Choong K (2017) Primary outcome measures in pediatric septic shock trials: a systematic review. Pediatr Crit Care Med 18:e146-e154

5. Merritt C, Menon K, Agus MSD, Choong K, McNally D, O'Hearn K, Watson RS, Wong HR, Duffett M, Wypij D, Zimmerman JJ (2018) Beyond survival: pediatric critical care interventional trial outcome measure preferences of families and healthcare professionals. Pediatr Crit Care Med 19:e105-e111

6. Morrison AL, Gillis J, O'Connell AJ, Schell DN, Dossetor DR, Mellis C (2002) Quality of life of survivors of pediatric intensive care. Pediatr Crit Care Med 3:1-5

7. Taylor A, Butt W, Chiardulli M (2003) The functional outcome and quality of life of children after admission to an intensive care unit. Intensive Care Med 29:795-800

8. Conlon NP, Breatnach C, O'Hare BP, Mannion DW, Lyons BJ (2009) Healthrelated quality of life after prolonged pediatric intensive care unit stay. Pediatr Crit Care Med 10:41-44

9. Polic B, Mestrovic J, Markic J, Mestrovic M, Capkun V, Utrobicic I, Jukica M, Radonic M (2013) Long-term quality of life of patients treated in paediatric intensive care unit. Eur J Pediatr 172:85-90

10. Colville GA, Pierce CM (2013) Children's self-reported quality of life after intensive care treatment. Pediatr Crit Care Med 14:e85-e92

11. Watson RS, Asaro LA, Hertzog JH, Sorce LR, Kachmar AG, Dervan LA, Angus DC, Wypij D, Curley MAQ, RESTORE Study Investigators and the PALISI Network (2018) Long-term outcomes after protocolized sedation versus usual care in ventilated pediatric patients. Am J Respir Crit Care Med 197:1457-1467

12. Kyosti E, Ala-Kokko T, Ohtonen P, Peltoniemi O, Rautiainen P, Kataja J, Ebeling $\mathrm{H}$, Liisanantti JH (2018) Factors associated with health-related quality of life six years after ICU discharge in a Finnish paediatric population: a cohort study. Intensive Care Med 44:1378-1387. https://doi.org/10.1007/ s00134-018-5296-4

13. Varni JW, Burwinkle TM, Seid M, Skarr D (2003) The PedsQL 4.0 as a pediatric population health measure: feasibility, reliability, and validity. Ambul Pediatr 3:329-341

14. Varni JW, Limbers CA, Burwinkle TM (2007) Impaired health-related quality of life in children and adolescents with chronic conditions: a comparative analysis of 10 disease clusters and 33 disease categories/severities utilizing the PedsQL 4.0 Generic Core Scales. Health Qual Life Outcomes $5: 43$

15. Simon TD, Cawthon ML, Stanford S, Popalisky J, Lyons D, Woodcox P Hood M, Chen AY, Mangione-Smith R, Center of Excellence on Quality of Care Measures for Children with Complex Needs (COE4CCN) Medical Complexity Working Group (2014) Pediatric Medical Complexity Algorithm: a new method to stratify children by medical complexity. Pediatrics 133:e1647-e1654 